\title{
Analytical science in Italy
}

\author{
Aldo Roda \\ Published online: 9 December 2012 \\ (C) Springer-Verlag Berlin Heidelberg 2012
}

Analytical chemistry is a truly interdisciplinary science, since it is involved in a variety of fields, ranging from chemistry disciplines (e.g., inorganic, organic, physical, pharmaceutical, and biological chemistry) to related sciences such as clinical chemistry, medicine, environmental science, agro food science, forensic science, cultural heritage science, space research, and geology. On the other hand, the development of new concepts in analytical and bioanalytical chemistry often requires and takes advantages of close collaborations among chemists and other scientists with different fields of expertise, from biologists to biotechnologists, bioinformatics, physicists, and engineers.

The present special issue entitled "Analytical Science in Italy" comprises more than 60 contributions (trends, critical reviews, original papers, and technical notes) representing, though not entirely, recent and updated achievements of the most established Italian research groups in analytical and bioanalytical sciences. Most of the articles were submitted by groups with different backgrounds and expertise working in universities throughout Italy.

The subjects of the contributions range from speciation studies to state-of-the-art instrumental analytical techniques, biosensors, and miniaturized bioanalytical devices. A large number of articles deal with high-performance separation methods, and focus on the development of innovative sample handling techniques as well as new analytical applications in biomedical, environmental, pharmaceutical, and forensic analysis. A variety of "omics" approaches offer modern solutions for the separation and detection of small molecules, proteins, and even whole cells. As could be predicted, because of the richness of Italian cultural heritage, several contributions report on new

Published in the special issue Analytical Science in Italy with guest editor Aldo Roda.

\section{A. Roda $(\triangle)$}

Department of Chemistry "G. Ciamician”, Alma Mater Studiorum,

University of Bologna, Via Selmi 2,

40126 Bologna, Italy

e-mail: aldo.roda@unibo.it analytical methods/techniques that focus on the characterization of inorganic and organic components in works of art. Finally, a number of contributions describe the application of nanoscience and new nanomaterials in bioanalytics for the development of nanodevices and miniaturized (bio)sensors based on electrochemical, optical, and luminescence signal transduction.

This special issue reflects the ability of the Italian analytical community to perform high-quality and often complementary research over a broad range of topics. Nevertheless, it represents only a glance at the large number of research groups working in Italian universities, public institutes, and private companies involved in the development and validation of analytical methods and instrumentation.

I wish to warmly thank Prof. Giuseppe Arena, President of the Analytical Chemistry Division of the Italian Chemical Society (Società Chimica Italiana), for promoting this initiative among the scientific community and Italian analytical chemists for their enthusiastic response to the invitation that led to the submission of a number of manuscripts that exceeded the most optimistic expectations. This clearly demonstrates that Analytical and Bioanalytical Chemistry is one of the reference journals for the whole Italian analytical chemistry community.

I hope that this special issue will contribute to creating new opportunities for international collaborations as well as to further strengthen the excellence of Analytical and Bioanalytical Chemistry.

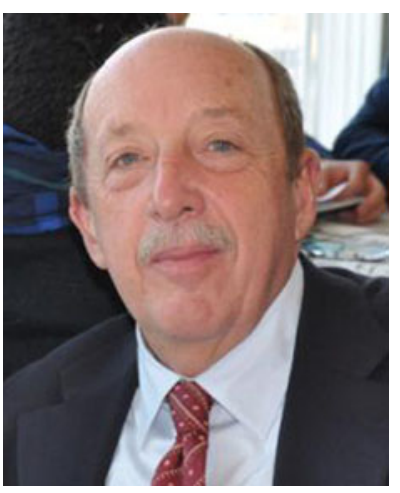

Aldo Roda is Professor of Analytical Chemistry at Bologna University. His main research interests center on the area of analytical and bioanalytical chemistry applied to clinical chemistry, pharmacotoxicology, medicinal chemistry, environmental and food analysis. He published more than 400 original articles on international journal and he is the owner of many patents on new bile acids drugs, new chemiluminescent and luciferases labels, and miniaturized devices. 